\title{
ReMed: rasche Hilfe
}

\author{
Das Unterstützungsnetzwerk ReMed berät und begleitet Ärztinnen und Ärzte \\ in kritischen Momenten - zum Beispiel dann, wenn der Berufsalltag zu einer \\ grossen Belastung wird.
}

\section{Jürg Beutler}

Kommunikation FMH

\section{Erfahrungsbericht}

Ein 45-jähriger Hausarzt mit eigener Praxis ist ganz unerwartet in eine Krise geraten.

«Ihnen geht es nicht gut», sagte mir eine Patientin nach der Begrüssung, als hätte sie selbst keine eigenen Sorgen. Und ein anderer Patient suchte sich einen neuen Hausarzt, da ich ihm nicht mehr belastbar schien. So augenfällig war die Krise, in der ich mich vor einiger Zeit befand.

Was war passiert? Eine Reise zu einem Freund, der seine Frau durch einen Verkehrsunfall verloren hatte und nun mit zwei kleinen Mädchen allein dastand, ging mir sehr nahe. Bei einem gemeinsamen Museumsbesuch brach die Krise urplötzlich über mich herein: Atemnot, Herzklopfen, Klaustrophobie. Fluchtartig musste ich das Gebäude verlassen. Meine Frau und mein Freund waren ebenso perplex wie ich.

Wieder zu Hause, schleppte ich mich mit letzter Energie durch Sprechstunde, Sitzungen und durch

ReMed meldet sich bei jeder Kontaktaufnahme innerhalb von 72 Stunden und bespricht unverbindlich und vertraulich die persönliche Situation und individuelle Handlungsmöglichkeiten. ReMed ist an das Arztgeheimnis gebunden.

Benötigen Sie Unterstützung? Oder eine Ärztin, ein Arzt aus Ihrem Umfeld?

Dann kontaktieren Sie ReMed:

24-Stunden-Hotline: 08000 73633,

info@swiss-remed.ch, www.swiss-remed.ch meine Einsätze als Bezirksarzt. Wie meine sensible Patientin gemerkt hatte, löste ihre Krankheit - und das war keine Ausnahme - bei mir panische Angst aus. In der Familie wich ich allen Verpflichtungen aus. Ich vernachlässigte Freunde, und allein das Klingeln des Telefons lösten Herzklopfen und Schweissausbrüche aus. Der Leidensdruck war so gross, dass ich schnell professionelle Hilfe in Anspruch nahm.

Der Weg aus der Krise war lang und anstrengend. Ich strukturierte meinen Alltag neu, gab Ämter ab und schuf leere Zeitfenster. Langsam lernte ich, dass ich nicht unentbehrlich bin und dass ich nicht allen Ansprüchen genügen kann. Letztlich hat sich diese Krise positiv auf alle Lebensbereiche ausgewirkt, besonders auf Partnerschaft und Familie.

\section{Interview mit Projektleiter}

Michael Peltenburg, was können Patientinnen und Patienten unternehmen, wenn sie merken, dass sich ihr Arzt in einer Krise befindet?

Ein Patient befindet sich in einer zwiespältigen Lage, wenn er seinen Arzt in Not sieht: Er möchte helfen, zugleich ist er auch verunsichert. Er spricht seinen Arzt deshalb nur selten darauf an. Vielleicht macht er eine Bemerkung zum Praxispersonal, das wahrt aber meist den Schein. Wenn die Situation unerträglich wird, wechselt er einfach den Arzt.

Doch der Patient kann sich jederzeit an ReMed wenden - namentlich oder anonym. ReMed tritt mit dem betroffenen Arzt in Kontakt und sucht das Gespräch. Verständlicherweise stösst dieser Schritt bei betroffenen Kollegen zunächst auf Misstrauen, doch letztlich wird die Unterstützung meist angenommen.

\section{Was unternimmt ReMed konkret?}

ReMed unterstützt den betroffenen Kollegen auf dem Weg aus der Krise. Er kann zum Beispiel eine Therapie, ein Coaching oder eine Beratung im Praxismanagement beginnen. ReMed hilft bei der richtigen Wahl und vermittelt Fachpersonen.

Und stellt ReMed auf Wunsch auch Kontakte zu Ärzten in gleichen Situationen her?

Wir wissen, dass eine Nachfrage nach Selbsthilfegruppen besteht. ReMed will auch in diesem Bereich einen aktiven Beitrag leisten. Wir möchten anregen, ermuntern - und Kontakte vermitteln. Wer sich angesprochen fühlt, soll sich bitte mit ReMed in Verbindung setzen. Konkret suchen wir in der Deutschschweiz Kolleginnen und Kollegen mit neurologischen Krankheiten mit ungewisser Prognose, die Interesse an einem Austausch von Copingstrategien haben.
Dr. med. Michael Peltenburg

Facharzt für Allgemeinmedizin

FMH

CH-8340 Hinwil

michael.peltenburg@hin.ch

\footnotetext{
S
}

